\title{
Isolation and Serological Analysis of Mutant Forms of Flagellar Antigen $i$ of Salmonella typhimurium
}

\author{
By T. M. JOYS* AND B. A. D. STOCKER \\ Guinness-Lister Research Unit, Lister Institute of Preventive \\ Medicine, London, S.W. 1
}

(Received 7 December 1965)

\begin{abstract}
SUMMARY
Nine spontaneous mutants with altered forms of flagellar antigen $i$ were obtained by picking more rapidly spreading swarms from growth in semi-solid medium containing enough anti-i serum to retard spreading growth. One mutant was in a line of Salmonella typhi given antigen $i$ by transduction, the rest in $S$. typhimurium strain LT2 adeC-7 proA-46. Two mutants of independent origin were serologically identical and presumably arose by a recurrence of the same mutation. Bacteria expressing the mutant phase-1 antigen were normally motile and the LT 2 mutants showed normal phase-variation, to give cultures with an apparently unaltered phase-2 antigen, 1,2,3. Flagellate bacteria with flagella of two of the mutant types, $i \mathbf{M} 6$ and $i \mathrm{M9}$, were agglutinated to titres 8-16 by sera from uninoculated rabbits and to titres 50-100 by sera from rabbits immunized with unrelated antigens; suspensions of flagella of these types, but not of others, caused flocculation of indian ink.

The residual activity of anti- $i$ (wild type) sera fully absorbed with mutant antigens showed that each mutant antigen had lost some of the serological specificity of the wild-type antigen; the complex pattern of residual activity on mutant and wild-type antigens of anti- $i$ (wild type) sera absorbed with pairs of mutant antigens indicated the existence of at least 13 antigenic factors in the wild-type antigen, and that each of the serologically distinct mutant antigens lacked a different combination of these factors. The residual activities on the homologous antigens of antimutant sera fully absorbed with wild-type antigen showed that all the mutant antigens, except perhaps $i \mathrm{M} 6$ and $i \mathrm{M} 9$, had antigenic specificities absent from the wild-type antigen. Each of the 8 serologically different antigens had a unique new specificity, but antigens $i \mathrm{M} 7, i \mathrm{M} 10$ and $i \mathrm{M} 12$ shared some new factors.

Attempts to infer the linear order of the presumed sites of amino acid substitution in the polypeptide chain of flagellin from the serological data were unsuccessful; this probably indicates the incorrectness of an assumption involved: namely, that anti-flagellar antibodies have an absolute affinity for, and only for, all of the amino acid side-chains (or all of a reactive subset of them) in a relevant length of polypeptide chain.
\end{abstract}

\section{INTRODUCTION}

The numerous phase 1 and phase 2 flagellar antigens of different salmonella serotypes or species, used for their classification in the Kauffmann-White table,

* Present address : Department of Bacteriology, University of Oregon Medical School, Portland, Oregon 97201, U.S.A. 
are determined by a series of allelic genes at two loci, $H 1$ and $H 2$ (Lederberg \& Edwards, 1953) which are widely separated on the linkage map (Smith \& Stocker, 1962; Mäkelä, 1964). The flagella of salmonellas are composed of a single protein, flagellin, probably made of a single polypeptide chain. It was therefore surmized that the serological differences between, for instance, phase-1 flagella of antigenic character $i$ and those of antigenic character $g, p$, reflected differences in the primary structure, that is the amino acid sequence of the two sorts of flagellin, determined by differences in the base sequence in the DNA of their structural genes, the $H 1$ alleles $H$ 1-i and $H$ 1-g, $p$. This assumption has been confirmed by the demonstration of considerable differences in amino acid composition between these two antigenically distinct salmonella flagellins (McDonough, 1962).

Variants of altered $\mathbf{H}$ antigenic constitution have been obtained from many salmonella species by growth in the presence of antiserum for the expressed $\mathbf{H}$ antigen(s), either growth in serum broth (Scott, 1926) or the more efficient technique of growth in semi-solid medium containing serum (Gard, 1937; Edwards \& Bruner, 1939 $a$; Bruner \& Edwards, 1939). Bacteria whose flagella combine with the antibody present are immobilized, and so grow only at the site of inoculation; whereas those whose flagella do not react remain motile and spread through the medium as they multiply. The presence of the antiserum thus permits detection of rare variants; there is no reason to suppose that it directly invokes them. In some of the many reported instances of changes in $\mathbf{H}$ antigenic constitution (Kauffmann, 1951; Joys, 1961) the new flagellar antigen obtained was a previously undetected alternate phase (Edwards \& Bruner, 1939b). In others the new antigen was a modified form of the original phase-1 or phase-2 antigen. Thus Gnosspelius (1939) obtained in Salmonella stanley $(4,5,12: d \leftrightarrow 1,2)$ a variant with a modified antigen $d$, and another with a modified antigen 1,2 , the other phase in each variant being unaltered.

We investigated spontaneous variation of the phase-1 antigen $i$ of Salmonella typhimurium strain LT2 $(4,5,12: i \leftrightarrow 1,2)$. In some previous investigations prolonged cultivation, sometimes with repeated subculture, in the presence of a high concentration of antibody has been used. Such methods presumably detect only variants with a considerably altered $\mathbf{H}$ antigen, perhaps resulting from the cumulative effect of more than one mutation. The commonest class of mutational alteration of flagellin which permitted normal locomotor function of the flagella would presumably be a single amino acid substitution. This would presumably cause only a minor change in serological character. To permit the detection of slightly altered forms of antigen and to minimize the risk of selecting double mutants, we used a concentration of anti- $i$ serum sufficient only to retard the spreading growth of bacteria expressing antigen $i$, and we picked faster-spreading 'swarms' after only 1 or 2 days of incubation. All of 8 such swarms tested proved to have modified forms of antigen $i$, as a result of spontaneous mutation at the $H 1$ locus. Preliminary accounts of the isolation, serology and genetics of the mutants (Joys \& Stocker, 1963) and of chemical investigations of their flagellins (McDonough, 1962) have been published. 


\section{METHODS}

Strains. A serologically mutated form of antigen $i$ was first encountered in an experiment with strain sw520, a derivative of Salmonella typhi strain Watson in which antigen $d$ had been replaced by antigen $i$, transduced by phage P22 from $S$. typhimurium strain LT7 (Zinder \& Lederberg, 1952). Serological mutants were later sought in strain Lт 2 adeC-7 pro $A-46$, a purine-exacting and proline-exacting double mutant of strain LT2. S. paratyphi $B$ O strain sw 543, a monophasic phase-1 strain lacking flagella through mutation at a locus co-transducible with $H 1$ (Stocker, Zinder \& Lederberg, 1953), was used as recipient when it was desired to transduce the $H 1$ locus of a serological mutant into a different genetic background.

Media and cultural methods. Nutrient broth and agar were made from a tryptic digest of meat. Large quantities of bacteria for absorbing sera were grown on a buffered peptone agar fortified with glycerol $(1 \%, \mathrm{v} / \mathrm{v})$, sodium lactate $(0.5 \%, \mathrm{w} / \mathrm{v})$ and Marmite autolysed yeast $(0.5 \%, w / v)$. A nutrient gelatin agar (Edwards \& Bruner, 1942; Stocker et al. 1953), which is semi-solid at $37^{\circ}$ but solid at room temperature, was used for the selection of highly motile bacteria and of serological mutants. Cultures were incubated at $37^{\circ}$; broth cultures were incubated unshaken.

Serological methods. Strains of bacteria to be used for preparation of agglutinable suspensions, absorbing suspensions or vaccines were first passaged through semisolid medium, to ensure good development of flagella. A discrete colony, shown by slide agglutination to be in the required $\mathbf{H}$ antigenic phase, was inoculated into broth; after incubation for a few hours formalin was added, to give a formaldehyde concentration of $0 \cdot 16 \%(\mathrm{w} / \mathrm{v})$ for agglutinable suspension, and $0 \cdot 8 \%(\mathrm{w} / \mathrm{v})$ for vaccines. Agglutinable suspensions were adjusted to match the turbidity of the standard suspensions of the Serological Standards Laboratory, Colindale, London, N.W. 9. Vaccines after sterility tests were inoculated intravenously into rabbits, four doses being given at 6-day intervals (Mackie \& McCartney, 1953). Sera were titrated by twofold steps, by the ordinary tube method or by a micromethod (Arkwright, 1927; Mandelbaum, 1932) to economize low-titre absorbed sera. In the micromethod a standard loopful (about $0.01 \mathrm{ml}$.) of agglutinable suspension as used for the tube tests was mixed with a standard loopful of a serum dilution on a glass plate which was incubated in a damp chamber $(16 \mathrm{~cm}$. Petri dish containing damp filter paper and cottonwool, enclosed in a plastic bag). After $2 \mathrm{hr}$ at $37^{\circ}$ and $1 \mathrm{hr}$ at room temperature, the droplets were examined for agglutination by naked eye and by low-power dark-ground microscopy. To avoid non-specific clumping due to the bacteria sticking to the glass, the plates were cleaned with chromic + sulphuric acid, rinsed in water and ethanol, dried with a fluff-free cloth and flamed before use. The micromethod was about one dilution more sensitive than the tube test. The titres by the micromethod were therefore recorded as the last positive serum dilution, disregarding the further approximate twofold dilution involved in mixing with the agglutinable suspension, so that titres recorded by the micro- and macro-methods were about the same.

For growth of absorbing suspensions, cultures of highly motile organisms were inoculated to nutrient agar in 11 . volumes in trays. Since the adenine-exacting strain gave rather poor growth on the medium used, derivative lines not exacting for adenine, obtained by transduction with phage P22, were generally used. 
After $48 \mathrm{hr}$ at $37^{\circ}$ the growth from a tray was harvested into $20 \mathrm{ml}$. saline with phenol $(0.5 \%, w / v)$, or of saline without phenol, because serum absorbed with phenol-killed bacteria was too toxic to use in semi-solid media for the selection of mutants. One volume of undiluted serum was mixed with 5 vol. of the dense unwashed suspension; after $2 \mathrm{hr}$ at $37^{\circ}$ the bacteria were centrifuged down. When the undiluted once-absorbed serum still agglutinated the absorbing strain this process was repeated once or more, using at each step equal volumes of absorbed serum and bacterial suspension, until no activity for the absorbing strain remained. When live bacteria had been used for absorption the fully absorbed serum was sterilized by Seitz filtration.

Serum number 713, used in isolation of the mutants, was an anti- $i$ serum prepared by immunization of a rabbit with a formalin-killed broth culture of a motile derivative of Salmonella paratyphi $B O$ strain sw 543, given antigen $i$ (wild type) from $S$. typhimurium strain LT2 by transduction with phage P22. Sera nos. 131 and 133 were from rabbits inoculated with a formalin-killed phase-1 culture of $S$. typhimurium LT2 adeC-7 proA-46, i.e. contained antibody evoked by wild-type antigen $i$. Serum GL 74, was an anti- $i$ serum received from the Serological Standards Laboratory, Central Public Health Laboratory, Colindale, London, N.W. 9.

\section{RESULTS}

\section{Isolation of mutants with altered antigen $i$}

A serologically altered form of antigen $i$ was first detected in an experiment in which salmonella strain sw 520 (Salmonella typhi given antigen $i$ by transduction) was inoculated into semi-solid medium containing anti- $i$ serum no. 713. A swarm spreading faster than the partly immobilized parent strain yielded a strain with a form of antigen $i$, labelled $i 2$, detectably different from the wild-type antigen $i$ of the parent and of strain LT2; for anti- $i$ serum no. 713, after complete absorption with antigen $i 2$, still agglutinated wild-type antigen $i$ to a titre of 400. Anti-i serum no. 713, unabsorbed or fully absorbed with $i 2$, was next used to isolate mutants with altered antigen $i$ from strain LT 2 adeC-7 pro $A$-46. Inocula from nine different single-colony stocks, previously passaged through semi-solid medium to enhance their motility, were stabbed into plates of semi-solid medium containing anti-1,2 serum, to immobilize bacteria in phase 2, and anti- $i$ serum, absorbed or unabsorbed, at a final concentration three times the agglutinating titre for wild-type $i$. At this concentration the growth of the inoculated strains spread slowly, taking about $60 \mathrm{hr}$ at $37^{\circ}$ to progress across a $5 \mathrm{~cm}$. plate. After incubation for 24 or $48 \mathrm{hr}$, two faster-spreading swarms were detected on plates containing unabsorbed serum and eight on plates containing absorbed serum (Table 1). These swarms, like the slow-spreading growth of the parent strains, had sharp edges, indicating that the flagellate bacteria of the swarm combined with, and were immobilized by, some antibody component in the anti- $i$ serum used (Stocker, 1956; Joys, 1961). The isolates from the ten faster-spreading swarms, labelled $\mathrm{m} 4$ to $\mathrm{m} 13$, all proved to have modified forms of antigen $i$, termed antigens $i$ M4-iM13. The serological and biochemical characters of isolate $\mathrm{m} 8$ showed that it was a re-isolate of the strain (S. typhi strain sw 520-i2) used to absorb serum 713, and evidently derived from a rare bacterium in the absorbing suspension which had survived contact with phenol. 
Strain $\mathrm{M} 8$ was included in the serological analysis, though it could not be used in later genetical experiments (Joys \& Stocker, 1963). Of the nine other isolates (all proven to have arisen from LT2) five (м6, м9, м10, м11, м12) clearly resulted from independent mutations, since each was isolated in a different single-colony stock. Isolate $\mathrm{m} 7$ was obtained by selection with unabsorbed serum from a stock which in a plate containing absorbed serum yielded $\mathbf{m} 4$ and $\mathbf{m} 5$; but since it differed serologically from them $\mathbf{m} 7$ must have resulted from an independent mutation.

\section{Table 1. Origin of the serological variants, and their agglutination}

by various anti-i (wild type) sera

$\begin{gathered}\text { Strain } \\ \text { Variants }\end{gathered}$
M 4
M 5
M 6
M 7
M 8
M 9
M 10
M 11
M 12
M 13
$i 2$

Parent strains

S. typhimurium LT 2 ade pro

S. typhi-i (sw 520)

\begin{tabular}{|c|c|c|c|c|c|}
\hline \multirow[b]{2}{*}{ Obtained from* } & \multirow{2}{*}{$\begin{array}{l}\text { Serum } \\
\text { used } \\
\text { to } \\
\text { select }\end{array}$} & \multicolumn{4}{|c|}{$\begin{array}{l}\text { Titre } \dagger \text { to which agglutinated } \\
\text { by anti- } i \text { serum no. }\end{array}$} \\
\hline & & 713 & $713 / i 2$ & 131 & 133 \\
\hline LT 2 ade pro $\mathbf{P 8}$ & $713 / i 2$ & 5,000 & 24 & 40,000 & 20,000 \\
\hline LT 2 ade pro P8 & $713 / i 2$ & 5,000 & 24 & 40,000 & 20,000 \\
\hline LT 2 ade pro $\mathrm{P} 4$ & 713 & 1,280 & 192 & 20,000 & 40,000 \\
\hline LT 2 ade pro P8 & 713 & 1,280 & 24 & 20,000 & 10,000 \\
\hline LT 2 ade pro $\mathbf{P 3} \ddagger$ & $713 / i 2$ & 5,000 & $<6$ & 40,000 & 20,000 \\
\hline LT 2 ade pro $\mathrm{P5}$ & $713 / i 2$ & $\mathbf{2 , 5 0 0}$ & 24 & 20,000 & $\mathbf{2 0 , 0 0 0}$ \\
\hline LT 2 ade pro $\mathbf{P 7}$ & $713 / i 2$ & 5,000 & 196 & 40,000 & 40,000 \\
\hline LT 2 ade pro $\mathbf{P 2}$ & $713 / i 2$ & 10,000 & 24 & 40,000 & 20,000 \\
\hline LT 2 ade pro P9 & $713 / i 2$ & 20,000 & 196 & 40,000 & 20,000 \\
\hline LT 2 ade pro P6 & $713 / i 2$ & 10,000 & $\mathbf{2 4}$ & 20,000 & 20,000 \\
\hline S. typhi-i (sw 520) & 713 & 5,000 & $<6$ & 40,000 & 20,000 \\
\hline & & 20,000 & 384 & 40,000 & 20,000 \\
\hline & . & 20,000 & 384 & . & . \\
\hline
\end{tabular}

* The variants were selected from single-colony isolates, P2-P9, of Salmonella typhimurium LT 2 adeC-7 pro $A-46$ by selection in semi-solid medium containing a just immobilizing amount of anti- $i$ serum no. 713, unabsorbed or fully absorbed with $S$. typhi-i2 (termed serum 713/i2).

$\dagger$ Titres by tube agglutination, except for serum $713 / i 2$, titrated by the micromethod; the titres of this serum (diluted $1 / 6$ in the course of absorbtion) are expressed in terms of content of original serum.

$\mp$ M 8 was isolated from a swarm on a plate inoculated with $L T 2$ ade pro $P 3$, but proved identical with $S$. typhi-i2, which had been used to make the absorbed serum incorporated in the medium.

Isolates м4 and м5, though picked from two apparently separate swarms on a single plate, were serologically identical; probably they were two isolates from a single mutant clone. Isolate $\mathrm{M} \mathbf{1 3}$ arose by mutation in a different single-colony stock but proved serologically identical with M4 (= M5); it presumably resulted from a recurrence of the mutation which produced m4 (= m5). The other seven isolates all differed serologically from $\mathrm{M} 4(=\mathrm{M} 5=\mathrm{M} 13)$ and from each other.

\section{Properties of serological mutants}

All the serological mutants were normally motile in phase 1 and the phase-1 suspensions were agglutinated to high titres by various anti- $i$ (wild type) sera (Table 1). All the LT2 mutants showed normal change of flagellar antigenic phase, to show an apparently unaltered phase-2 antigen, 1,2,3. Subsequent serological and chemical examination showed that none of them were $n m l^{-}$mutants, with the (serologically distinguishable) flagella which lack the $\epsilon-N$-methyllysine which is 
present in the flagella of wild-type Salmonella typhimurium (Stocker, McDonough \& Ambler, 1961; Mrs U. B. Pearce, T. M. Joys \& B. A. D. Stocker, unpublished). All the mutants gave stable $H$ suspensions; but the phase-1 suspensions of $\mathbf{m} 6$ and $\mathrm{m} 9$ showed an abnormal reactivity with some 'non-specific' reagents.

Special properties of $\boldsymbol{M}_{6}$ and ${ }_{\mathrm{M}} 9$. Formalinized phase-1 suspensions of $\mathrm{m} 6$ and $\mathrm{m} 9$, but not their phase-2 suspensions nor phase-1 suspensions of the other mutants, were agglutinated by all of ten sera from uninoculated rabbits, to titres of 8-16; washed deflagellated $\mathrm{m} 6$ and $\mathrm{m} 9$ phase- 1 suspensions were unaffected.The $i \mathrm{M} 6$ and $i \mathrm{M} 9$ suspensions were likewise agglutinated, to titres of 50-100, by four sera from rabbits inoculated with salmonellas having no $\mathrm{O}$ or phase-1 $\mathrm{H}$ antigens in common with Salmonella typhimurium; a wild-type $i$ suspension was not agglutinated by these sera, even undiluted. Salmonella paratyphi $B$ sw 543 transductants given antigens $i \mathrm{M} 6$ or $i \mathrm{M} 9$ were similarly agglutinated by 'non-specific' sera; transductants given antigen $i$ (wild type) or $i \mathrm{M} 10$ were unaffected. Evidently the phase-1 flagella are the site of the abnormal agglutinability of mutants м6 and м9. In experiments on the agglutination of suspensions of detached flagella by sera we added indian ink to enhance the visibility of the flocculated flagella (Craigie, 1931). When 10 vol. of diluted indian ink (3 drops of ink in $5 \mathrm{ml}$. saline) and 1 vol. of $i \mathrm{M} 6$ or $i \mathrm{M} 9$ flagellar suspensions were mixed, large black granules rapidly formed; flagella from the $i \mathrm{M} 6$ and $i \mathrm{M} 9 S$. paratyphi $B$ transductants had the same effect. Even tenfold higher concentrations of $i$ (wild type) flagella or flagella of the other mutant- $i$ types did not cause such flocculation. The flocculation of ink by $i \mathbf{M} 6$ and $i \mathbf{M} 9$ flagella was obtained only at $\mathrm{NaCl}$ concentrations $>0.3 \%(\mathrm{w} / \mathrm{v}) ;>1 \% \mathrm{NaCl}$ caused flocculation of the ink even in the absence of flagella. The antigenic analysis of $i \mathrm{M} 6$ and $i \mathrm{M} 9$ was complicated by their agglutinability by non-specific components of anti- $i$ sera-that is, components not able to combine with the immunizing antigen. Thus anti- $i$ (wild type) serum no. 131 and anti-iM10 serum no. 57 after complete absorption with their homologous antigens still agglutinated $i \mathrm{M} 6$ and $i \mathrm{M9}$ suspensions, despite dilution to $1 / 24$ and $1 / 12$, respectively, in the course of absorption. As $i$ M6 and $i$ M9 flagella were alike in their reaction with indian ink and with components present in the serum of uninoculated rabbits (and, in higher concentrations, in sera of rabbits immunized with related or unrelated antigens) it was expected that the same normal serum component reacted with both $i \mathbf{M} 6$ and $i$ M9. However, a sample of serum from an uninoculated rabbit after complete absorption with $i$ M 6 still agglutinated $i \mathrm{M} 9$; and a sample fully absorbed with $i \mathrm{M} 9$ still agglutinated $i \mathrm{M} 6$.

\section{Nerw serological specificities of mutant antigens}

Two rabbits were immunized with antigen $i \mathrm{M} 6$, two with $i \mathrm{M} 12$ and one each with $i \mathrm{M} 5, i \mathrm{M} 7, i \mathrm{M} 8, i \mathrm{M} 9, i \mathrm{M} 10, i \mathrm{M} 11$ and $i \mathrm{M} 13 ; i \mathrm{M} 4$ and $i 2$ were not used, being regarded as identical with $i \mathrm{M} 5$ and $i \mathrm{M} 8$, respectively. All the anti-mutant sera obtained agglutinated the homologous antigens to titres of 10,000-40,000, except the two anti-iM6 sera, which agglutinated the homologous suspensions to titres of only 1000-3000 (Table 2). All the sera agglutinated wild-type $i$ and the remaining antigens to about the same titre as the immunizing antigen. Since the unabsorbed anti-mutant sera did not distinguish their homologous antigens from the wild-type and the other mutant antigens, each anti-mutant serum was fully absorbed with the wild-type antigen. Each of the fully absorbed anti-mutant sera still agglutinated 
its homologous antigen, to titres of between 200 (anti-iM5 serum no. 48) and 6000 (anti-iM 8 serum no. 85). The absorbed sera did not agglutinate salmonella $\mathbf{H}$ suspensions representing the following phase-1 antigens: $a ; b ; c ; d ; e, h ; e, n, x ; g, p$; and $r$. The two anti-iM6 sera and the anti-iM9 serum after absorption with wildtype antigen had titres on their homologous antigens of only 200-400, not much greater than the titres of 50-100 on the same suspensions of sera from rabbits immunized with antigenically unrelated salmonellas. With this exception, the results

Table 2. Log titres (to base 2) on mutant antigens of anti-mutant sera fully absorbed with wild-type antigen

\section{Serum}

anti-M 5 no. 48 anti-M 13 no. 58

(anti-M 6 no. 55

anti-M 6 no. 132

anti-M 9 no. 56

anti-M 7 no. 84

anti-M 10 no. 57

(anti-M 12 no. 128

anti-M 12 no. 22

anti-M 8 no. 85

anti-M 11 no. 86

anti- $i$ (wild-type)

no. 131

\begin{tabular}{|c|c|c|c|c|c|c|c|c|c|c|}
\hline \multirow{2}{*}{$\begin{array}{c}\text { Titre* } \\
\text { before } \\
\text { absorption }\end{array}$} & \multirow{2}{*}{$\begin{array}{c}\text { Dilution } \\
\text { during } \\
\text { absorption }\end{array}$} & \multicolumn{9}{|c|}{ Log titre of absorbed serum on } \\
\hline & & $\mathbf{M 5}+$ & M 13 & $\mathbf{M} 6$ & $\mathbf{M} 9$ & M7 & M 10 & M 12 & M $8 \ddagger$ & $\mathbf{M} 1$ \\
\hline 10,000 & $1 / 48$ & 2 & 2 & . & . & . & . & . & . & - \\
\hline 20,000 & $\mathbf{1} / \mathbf{2 4}$ & 4 & 4 & 1 & $\mathbf{2}$ & - & . & - & . & - \\
\hline 1,000 & $1 / 12$ & . & . & 5 & 4 & - & . & . & . & $\cdot$ \\
\hline 3,000 & $1 / 12$ & . & . & 4 & 4 & . & . & • & . & $\cdot$ \\
\hline 10,000 & $1 / 12$ & . & . & 4 & 4 & . & . & . & . & . \\
\hline 10,000 & $1 / 24$ & - & . & 4 & 3 & 8 & 6 & 5 & . & . \\
\hline 40,000 & $1 / 24$ & . & . & 3 & 3 & 1 & 6 & 2 & . & . \\
\hline 10,000 & $1 / 24$ & . & . & 4 & 2 & . & 1 & 6 & . & . \\
\hline 10,000 & $1 / 12$ & . & . & $\mathbf{3}$ & $\mathbf{3}$ & 1 & 2 & 7 & . & . \\
\hline 20,000 & $1 / 24$ & . & . & 2 & $\mathbf{3}$ & . & . & . & 8 & \\
\hline 10,000 & $1 / 24$ & . & . & 1 & 1 & . & . & . & . & 5 \\
\hline 20,000 & $1 / 24$ & . & . & 3 & 2 & . & . & . & . & . \\
\hline
\end{tabular}

The anti-sera after complete absorption with wild-type antigen were tested by the micromethod on the indicated antigens. Titres are stated as last effective dilution, expressed as $\log$ to base 2 , of the absorbed antiserum itself; titres in terms of content of original antiserum can be calculated from dilution involved in absorption (col. 3). Bold-face figure = titre on homologous antigen. .$=$ no agglutination in undiluted absorbed antiserum. Antigens arranged in an order which brings together mutants with cross-reactive mutant specificities (м 5 and $м 13$; 6 and $м 9$; м 7, м 10 and м 12). The last antiserum listed is an anti-i (wild type) serum, illustrating the agglutination of $i \mathrm{M6}$ and $i \mathrm{M9}$ suspensions by sera fully absorbed with their homologous antigens.

* Titre on homologous antigen, wild-type antigen and most non-homologous mutant antigens.

$\dagger$ A suspension of $M 4$, which is probably the same mutant as $M 5$, gave the same results as the M 5 suspension with all sera.

$\ddagger$ A suspension of $S$. typhi-i2, which is probably the same strain as $\mathrm{M} 8$, gave the same results as the $\mathrm{m} 8$ suspensions with all sera.

indicate that each mutant antigen has a specificity or antigenic subfactor absent in wild-type antigen $i$. Some of the absorbed anti-mutant sera agglutinated mutant antigens other than their homologous antigen. We attribute the agglutination of the $i \mathrm{M} 6$ and $i \mathrm{M} 9$ suspensions by nearly all the absorbed anti-mutant sera, to titres of 50-400, to the 'non-specific' agglutinability of these antigens by unrelated immune sera, discussed above. The other cross-reactions, presumably indicative of antigenic relatedness of the mutant specificities of the mutants concerned, were investigated by cross-absorption.

Cross-reactions of $i M 4, i M 5$ and $i M 13$. These three suspensions were agglutinated to equal titres by the absorbed anti-iM5 serum no. 48 and also by the absorbed anti-iM13 serum no. 58. To test the antigenic relationship of $i$ M5 and $i$ M13, known to be of independent origin, unabsorbed anti- $i \mathrm{M} 13$ serum was absorbed with $i$ M5 and unabsorbed anti-iM5 serum with $i$ M13. With each successive absorption 
the titres of each serum on $i \mathrm{M} 13$ and $i \mathrm{M} 5$ decreased pari-passu, and the fully cross-absorbed sera did not agglutinate their homologous suspensions. Thus the mutant specificity of $i \mathrm{Ml3}$ was indistinguishable from that of $i \mathrm{M} 5$ by the 'mirror' absorption test; this and the similar activities of anti- $i$ (wild-type) sera absorbed with $i \mathrm{M} 5$ and $i \mathrm{M} 13$ show that these two antigens are serologically identical. (The $i \mathrm{M} 4$ suspension was agglutinated to the same titres as the $i$ M 5 and $i$ M13 suspensions at each stage, as expected in view of the probable origin of $M 4$ from the same mutant clone as $\mathrm{M} 5$.)

$i M 8$ and $i 2$. The agglutination of the Salmonella typhi- $i 2$ suspension by anti-iM8 serum absorbed by wild-type antigen $i$ is accounted for by the identity of $\mathrm{m} 8$ and $S$. typhi-i2, noted above.

$i M 7, i M 10$ and $i M 12$. The fully absorbed sera against these mutant antigens agglutinated all three of them, in each case the homologous antigen to a higher titre than the two heterologous antigens. Appropriate cross-absorption tests were made. If an antigenic subfactor is postulated to account for each distinct type of antibody recognized by the reactions of the cross-absorbed sera, the results indicate the presence of mutant subfactors as follows:

$$
\begin{aligned}
& i \mathrm{M7}=a \quad b \quad c-\overline{-}- \\
& i \mathrm{M} 10=a \quad b-d e- \\
& i \mathrm{M} 12=a--d-f
\end{aligned}
$$

\section{Absorption of anti-i (wild-type) sera by mutant antigens}

Several anti- $i$ (wild-type) sera were titrated on wild-type and all the mutant antigens (Table 1). As was expected antisera 713 and 713 absorbed with $i 2$ (the sera used in selection of the mutants) agglutinated nearly all the mutant suspensions to lower titres than the wild-type antigen; so also did one other anti- $i$ (wild-type) serum tested later (no. GL74). Two other anti- $i$ sera (nos. 131, 133) had, however, about equal titres on all the mutant and the wild-type antigens. Several anti- $i$ (wild-type) sera were next absorbed with each serological mutant, and tested for activity on wild-type antigen, to look for subfactors present in the wild-type antigen but absent from the mutants. Samples of three sera, nos. 131, 133 and GL74, were fully absorbed with each of the mutant antigens, $i \mathrm{M} 4$ to $i \mathrm{M} 13$ and $i 2$; serum no. 713 because of its scarcity was absorbed only with $i 2, i \mathrm{M} 9, i \mathrm{M} 10$ and $i$ M13. The absorbed samples, diluted to $1 / 12-1 / 48$ in the course of absorption, were tested on suspensions of wild-type $i$ and non-homologous mutant antigens by the micromethod and titrated on those suspensions which were agglutinated. The titres are recorded in Table 3 as logs to the base 2 of the dilutions of the absorbed sera. Complete absorption of any of the four sera with any mutant antigen except $i$ M 12 left titres of at least $1 / 32$ of the absorbed serum for wild-type $i$, and variable titres for some of the heterologous mutant antigens. After absorption with $i \mathrm{M} 12$ only a low titre for wild-type $i$ remained. Thus each mutant antigen did not absorb some species of antibody active on the wild-type antigen, and so was inferred to have lost some antigenic specificity.

Each of the serum samples, unabsorbed or absorbed, had equal titres on the three suspensions $i \mathrm{M} 4, i \mathrm{M} 5$ and $i \mathrm{M13}$, later found to be serologically identical; but all the other suspensions differed in their agglutinability. Correspondingly, each serum had 
an essentially similar spectrum of residual activity after absorption with either $i \mathrm{M} 4$ or $i \mathrm{M} 5$ or $i \mathrm{M13}$; whereas absorption with each of the other mutants revealed a unique spectrum of residual activity. Thus all the mutant antigens except these three differed from each other. The four anti- $i$ sera after similar absorptions had

Table 3. Log titres (to base 2) on wild-type and mutant antigens of several anti-i (wild type) sera fully absorbed with different mutant antigens

\begin{tabular}{|c|c|c|c|c|c|c|c|c|c|c|c|c|}
\hline \multirow{2}{*}{$\begin{array}{c}\text { Serum } \\
\text { no. }\end{array}$} & \multirow{2}{*}{$\begin{array}{l}\text { Absorbed } \\
\text { with }\end{array}$} & \multirow{2}{*}{$\begin{array}{l}\text { Dilution } \\
\text { during } \\
\text { absorp- } \\
\text { tion }\end{array}$} & \multicolumn{10}{|c|}{ Log titre of absorbed serum on } \\
\hline & & & $i$ (w.t.) & M5* & M13 & M6 & M9 & м7 & M 10 & M 12 & M 8 & M11 \\
\hline 131 & M 4 & $1 / 24$ & 9 & $<\mathbf{0}$ & $<0$ & 8 & $\mathbf{3}$ & $<0$ & 6 & 9 & 4 & 4 \\
\hline 133 & M 4 & $1 / 24$ & 8 & $<\mathbf{0}$ & $<0$ & 6 & 2 & $<0$ & $\mathbf{5}$ & 6 & $\mathbf{3}$ & $\mathbf{2}$ \\
\hline GL $\quad 74$ & $\mathbf{M} 4$ & $1 / 12$ & 6 & $<\mathbf{0}$ & $<0$ & 4 & $\mathbf{0}$ & $<0$ & 3 & 4 & 2 & $\mathbf{0}$ \\
\hline 131 & M 5 & $1 / 24$ & 9 & $<0$ & $<0$ & 9 & 1 & $<0$ & 6 & 9 & 4 & 1 \\
\hline 133 & M 5 & $1 / 24$ & 8 & $<\mathbf{0}$ & $<0$ & 7 & 3 & $<0$ & 6 & 7 & 3 & 2 \\
\hline GL 74 & M 5 & $1 / 12$ & 6 & $<0$ & $<0$ & 4 & $\mathbf{0}$ & $<0$ & 2 & 4 & 4 & 0 \\
\hline 131 & M 13 & $1 / 24$ & 9 & $<0$ & $<0$ & 9 & $\mathbf{0}$ & $<0$ & 7 & 9 & 5 & 4 \\
\hline 133 & M 13 & $1 / 24$ & 8 & $<0$ & $<0$ & 7 & 2 & $<0$ & 5 & 7 & 3 & 2 \\
\hline GL 74 & м 13 & $1 / 12$ & 6 & $<0$ & $<0$ & 4 & $\mathbf{0}$ & $<0$ & $\mathbf{3}$ & 4 & 0 & o \\
\hline 713 & M 13 & $1 / 24$ & 10 & $<0$ & $<0$ & 10 & 5 & $<0$ & 9 & 10 & 7 & 2 \\
\hline 131 & м 6 & $1 / 24$ & 10 & 10 & 10 & $<0$ & $<0$ & 9 & 10 & 10 & 10 & 10 \\
\hline 133 & M 6 & $1 / 24$ & 8 & 7 & 7 & $<0$ & $<0$ & 7 & 7 & $\gamma$ & 7 & 7 \\
\hline GL $\quad 74$ & M 6 & $1 / 12$ & 7 & 6 & 6 & $<0$ & $<0$ & 6 & 6 & 6 & 6 & 6 \\
\hline 131 & м 9 & $1 / 12$ & 10 & 10 & 10 & 10 & $<0$ & 10 & 10 & 10 & 10 & 10 \\
\hline 133 & M 9 & $1 / 12$ & 9 & 9 & 9 & 9 & $<0$ & 9 & 9 & 9 & 9 & 9 \\
\hline GL $\quad 74$ & M 9 & $1 / 12$ & 8 & 6 & 6 & 6 & $<0$ & 6 & 6 & 6 & 6 & 6 \\
\hline$\gamma 13$ & M 9 & $1 / 24$ & 10 & 9 & 9 & 10 & $<0$ & 6 & 9 & 9 & 9 & 9 \\
\hline 131 & M 7 & $1 / 12$ & 10 & 8 & 8 & 10 & $\mathbf{5}$ & $<0$ & 9 & 10 & 8 & 9 \\
\hline 133 & $\mathbf{M} 7$ & $1 / 12$ & 10 & 6 & 6 & $\mathbf{9}$ & 5 & $<0$ & 7 & 9 & 6 & 6 \\
\hline GL 74 & M 7 & $1 / 12$ & 7 & 4 & 4 & 6 & o & $<\mathbf{0}$ & 5 & 6 & 4 & 5 \\
\hline 131 & M 10 & $1 / 48$ & 6 & 5 & 5 & 6 & 2 & $<0$ & $<0$ & 6 & 3 & 4 \\
\hline 133 & M 10 & $1 / 24$ & 6 & 5 & $\mathbf{5}$ & 5 & 2 & $<0$ & $<0$ & 5 & 2 & $\mathbf{5}$ \\
\hline GL 74 & M 10 & $1 / 12$ & $\mathbf{5}$ & 3 & 3 & 3 & 2 & $<0$ & $<0$ & $\mathbf{3}$ & 2 & $\mathbf{3}$ \\
\hline 713 & M 10 & $1 / 48$ & 7 & 0 & $\mathbf{0}$ & 4 & 3 & $<0$ & $<0$ & 7 & $\mathbf{3}$ & $\mathbf{3}$ \\
\hline 131 & M 12 & $1 / 48$ & 1 & $<0$ & $<0$ & $<0$ & $<0$ & $<0$ & $<0$ & $<0$ & $<0$ & $<0$ \\
\hline 133 & M 12 & $1 / 24$ & 1 & $<0$ & $<0$ & $<0$ & $<0$ & $<0$ & $<0$ & $<0$ & $<\mathbf{0}$ & $<0$ \\
\hline GL 74 & M 12 & $1 / 12$ & 3 & 3 & 3 & $<0$ & $<0$ & $<0$ & $<0$ & $<0$ & $<0$ & $<0$ \\
\hline 131 & M 8 & $1 / 12$ & 9 & 7 & 7 & 9 & 6 & 6 & 9 & 9 & $<0$ & 6 \\
\hline 133 & M 8 & $1 / 12$ & 8 & 6 & 6 & 7 & 5 & $\mathbf{5}$ & 7 & 7 & $<0$ & 6 \\
\hline GI 74 & M 8 & $1 / 12$ & 6 & 2 & 2 & 4 & 1 & 1 & 4 & 4 & $<0$ & 2 \\
\hline 713 & $i 2(=\mathrm{M} 8)$ & $1 / 6$ & 7 & $\mathbf{3}$ & $\mathbf{3}$ & 6 & $\mathbf{3}$ & 3 & 6 & 6 & $<\mathbf{0}$ & 3 \\
\hline 131 & M 11 & $1 / 24$ & 9 & 6 & 6 & 9 & 4 & 1 & 8 & 9 & 8 & $<0$ \\
\hline 133 & M 11 & $1 / 24$ & 8 & 3 & 3 & 7 & 3 & $<0$ & 6 & 7 & 5 & $<0$ \\
\hline GL 74 & M 11 & $1 / 12$ & 7 & 2 & 2 & $\mathbf{5}$ & 1 & $<0$ & $\mathbf{3}$ & 5 & 2 & $<\mathbf{0}$ \\
\hline
\end{tabular}

The antisera before absorption had titres on $i$ (wild type) of 40,000 (no. 131), 20,000 (no. 133), 10,000 (no. GL 74) and 20,000 (no. 713); the mutant antigens were agglutinated to about the same titres, except by antiserum 713, which had lower titres on most of them (Table 1). The antisera after complete absorption with the indicated antigen were tested by the micromethod. Their titres are stated as last effective dilution, expressed as log to base 2, of the absorbed antiserum itself; titres in terms of content of original serum can be calculated from dilution involved in absorption (col. 3).

$0=$ agglutination only in undiluted absorbed antiserum. $<0=$ no agglutination in undiluted absorbed antiserum. $<0=$ negative reaction with antigen used for absorption.

* A suspension of $\mathrm{m} 4$, which is probably the same mutant as $\mathrm{m} 5$, gave the same results as the 5 suspension with all sera. 
patterns of residual activity which were qualitatively similar though not quantitatively identical. For instance after complete absorption with $i$ M13 all four sera had a titre on $i \mathrm{M} 6=$ titre on $i \mathrm{M} 12>$ titre on $i \mathrm{M} 10>$ titre on $i \mathrm{M} 8$; and did not agglutinate $i \mathbf{M r}$.

Double absorptions of anti-i (wild type) serum. The complex pattern of activity on heterologous mutant antigens of the absorbed samples of anti- $i$ (wild type) sera suggested that the wild-type antigen $i$ was complex. One anti- $i$ (wild type) serum, no. 133, was therefore fully absorbed with all possible pairs of the eight serologically different mutant antigens, the serologically identical set $i \mathrm{M} 4, i \mathrm{M} 5$ and $i \mathrm{M} 13$ being represented by $i$ M 4 alone. Each double absorption was made in two different orders, e.g. a sample absorbed with $i \mathrm{M} 4$ was further absorbed with $i \mathrm{M} 6$, and a sample absorbed with $i \mathrm{M} 6$ was further absorbed with $i$ M4. The doubly absorbed sera, diluted to 1/12-1/96 in the course of absorption, were tested without further dilution by the micromethod on all the $i$ antigens. In every test the two different samples of doubly absorbed serum behaved alike (Table 4). All the doubly absorbed sera still agglutinated the wild-type antigen, but they differed widely in their activities on the mutant antigens not used for absorption. Thus, some agglutinated none of the mutant antigens, e.g. serum absorbed with $i \mathrm{M} 6$ and $i \mathrm{M} 8$; whereas others, for instance that absorbed with $i \mathrm{M} 4$ and $i \mathrm{M} 7$, agglutinated all the mutant antigens except the two absorbing antigens. We did not proceed to further absorptions of those doubly absorbed sera which still agglutinated more than one antigen. Partly for this reason it was difficult to analyse the data in the ordinary way, postulating antigenic subfactors in the wild-type antigen, each defined by its reaction with a particular species of antibody left as the sole antibody remaining after single, double, triple, etc. absorption. However, we attempted to find the distribution in the mutants of the minimum number of subfactors to account for the activities of the singly and doubly absorbed samples. The scheme arrived at (Table 5) is based on the assumption that the unabsorbed anti- $i$ serum no. 133 contains antibodies specific for each of the thirteen postulated subfactors of antigen $i$ and that after single or double absorption it will still agglutinate all mutant antigens containing a given factor if the absorbing suspension(s) lacked that factor.

The full argument leading to the scheme is too lengthy to reproduce here, but may be illustrated as follows. Consider those absorbed samples which agglutinate only the wild-type antigen, i.e. antiserum absorbed with $i \mathrm{M} 12$ alone, or with $i \mathrm{M} 4+$ $i \mathrm{M} 6$, or with $i \mathrm{M} 6+i \mathrm{M} 8$, etc. We ascribe their activity to residual antibody for a factor, G, present in the wild-type antigen and absent from all the mutant antigens. Consider next the absorbed samples with the next most restricted range, those absorbed with $i \mathrm{M} 4+i \mathrm{M} 10$, and with $i \mathrm{M} 10+i \mathrm{M} 11$, which agglutinate only $i \mathrm{M} 6$, $i \mathrm{M} 12$ and wild-type $i$. We postulate a factor, J, present in $i \mathrm{M} 6$ and $i \mathrm{M} 12$, but absent in $i \mathrm{M} 4, i \mathrm{M} 10$ and $i \mathrm{M} 11$ (since absorption with them did not remove the anti-J activity), and absent also from the remaining mutant antigens because they were not agglutinated by the doubly absorbed antisera having anti-J activity. Since absorption with the wild-type antigen removed all activity from the antiserum this also must possess factor $\mathbf{J}$-and, by the same argument, all the other factors postulated. (The doubly absorbed antisera had been diluted during absorption beyond the range of activity of the non-specific agglutinins for $i \mathbf{M} 6$ and $i \mathbf{M 9} 9$ ). If, as postulated, all the mutant antigens except $i \mathrm{M} 6$ and $i \mathrm{M} 12$ lacked factor $\mathrm{J}$, 


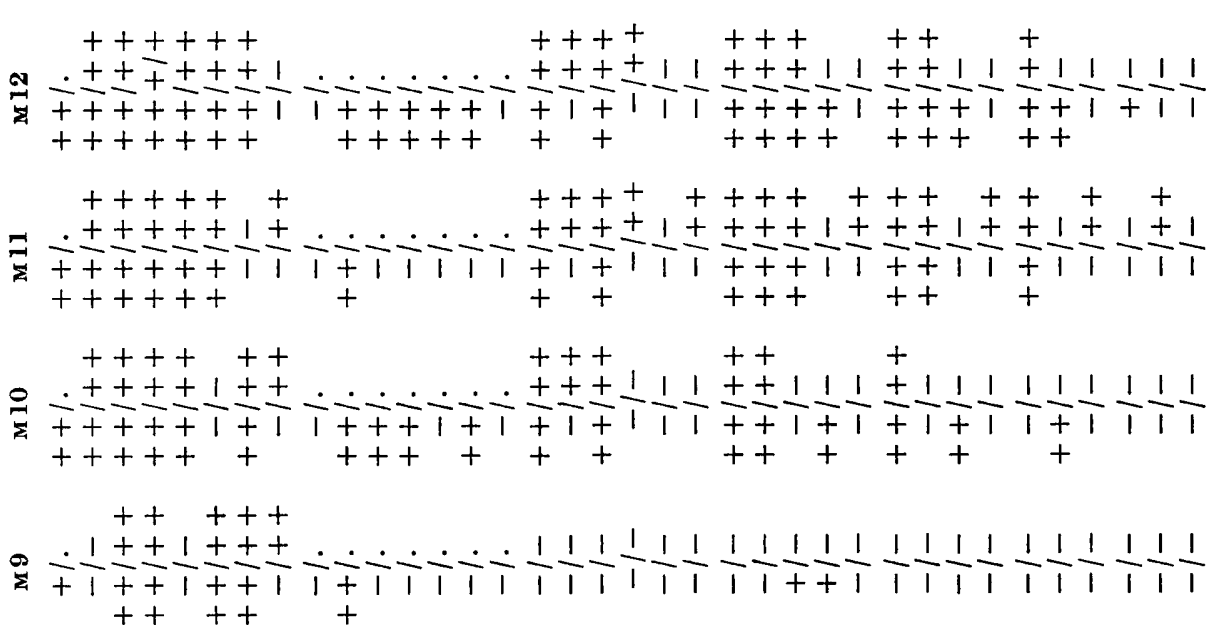

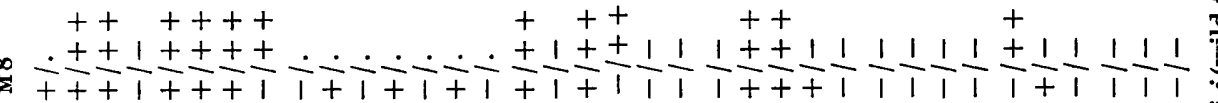
$++++++++++$

$++$

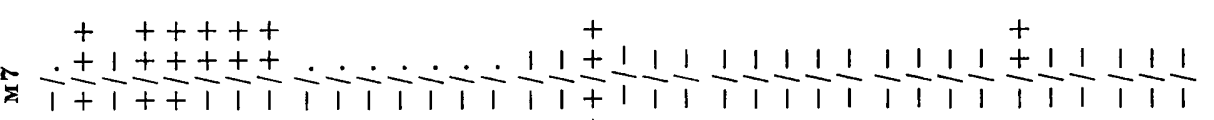
$+++$

$+++++$

$\therefore \dot{1+\div \div} \div 111111 \pm+1$

$1111 \pm$

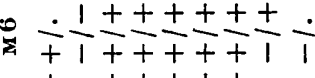

11 i

$+++++$

$++++$

++++
+++

$++$

$++++++$

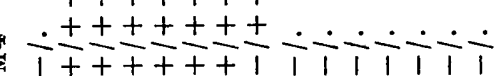

$++++++++++++++++++$ $+++t+++++++++++++++++$

$+++++$

$$
++
$$

++++
+++

$++$

$++$

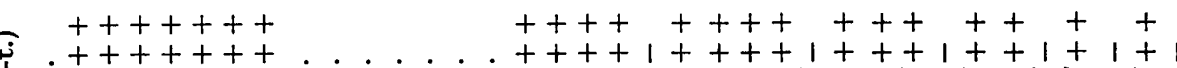

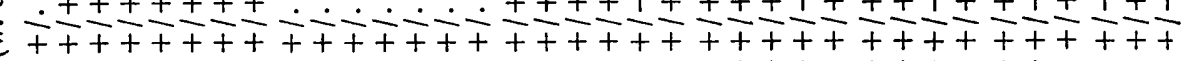
$+++++++++++++++++++++$

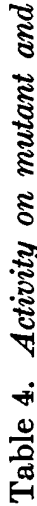

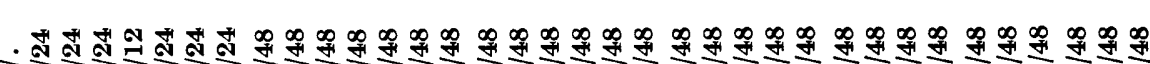
ה

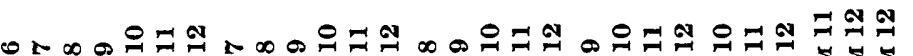


then antiserum absorbed with any mutant or pair of mutants, not including either of these two, should still contain anti-J and should agglutinate $i \mathrm{M} 6$ and $i \mathrm{M12}$; as was observed (Tables 3 and 4). Now consider the absorbed samples with the next most restricted activity, those absorbed with $i \mathbf{M} 4+i \mathbf{M} 8$, and with $i \mathbf{M} 8+i \mathbf{M} 11$, which agglutinate $i \mathrm{M} 6, i \mathrm{M} 10$ and $i \mathrm{M12}$, as well as wild type. The activity of these samples on $i \mathrm{M} 6$ and $i \mathrm{M} 12$ is accounted for by the presence of anti-J antibody. To account for the activity on $i \mathrm{M} 10$ we postulate a further factor $\mathrm{H}$, present in $i \mathrm{M} 10$,

Table 5. Distribution in mutant antigens of subfactors of antigen $i$ (wild type) inferred from activities of singly and doubly absorbed samples of anti-i (wild type) antiserum no. 133 and anti-iM 13 serum no. 58

\begin{tabular}{|c|c|c|c|c|c|c|c|c|c|c|c|c|c|c|c|c|c|c|}
\hline \multirow{2}{*}{$\begin{array}{l}\text { Antigen } \\
\mathrm{i} \text { (w.-t.) }\end{array}$} & \multicolumn{18}{|c|}{ Factors of $i$ (wild-type) antigen } \\
\hline & B & C & D & $\mathbf{E}$ & $\mathbf{F}$ & G $\mathbf{H}$ & I J & $\mathbf{K}$ & $\mathbf{L} \mathbf{M}$ & $1 \mathrm{~N}$ & $\mathbf{O}$ & $\mathbf{P} \mathbf{Q}$ & $R$ & $\mathbf{S}$ & $\mathbf{T} \mathbf{C}$ & $\mathbf{U}$ & $\mathbf{v} \mathbf{v}$ & $\mathbf{w} \mathbf{x}$ \\
\hline$i \mathrm{M} 4$ and $i \mathrm{M} 13$ & B & C & . & $\mathbf{E}$ & $\mathbf{F}$ & . & & $\mathbf{K}$ & $\mathbf{L} \mathbf{M}$ & & $\mathbf{O}$ & $\mathbf{P} \mathbf{Q}$ & $\mathbf{R}$ & S T & $\mathbf{T} \mathbf{L}$ & $\mathbf{U}$ & $\mathbf{V} \mathrm{V}$ & $\mathbf{w} \mathbf{x}$ \\
\hline$i \mathrm{M} 6$ & & $\mathbf{C}$ & D & $\mathbf{E}$ & $\mathbf{F}$ & . $\mathbf{H}$ & $\mathbf{J}$ & . & L $\mathbf{M}$ & I $\mathrm{N}$ & 0 & $\mathbf{P} \mathbf{Q}$ & $R$ & . & . & . & & . $\mathbf{x}$ \\
\hline iM7 & $\mathbf{B}$ & C & . & & . & . . & . & . & . . & . & . & $\mathbf{P} \mathbf{Q}$ & $R$ & . & . & . & . & . $\mathbf{x}$ \\
\hline$i \mathrm{M} 8$ & B & . & D & $\mathbf{E}$ & $\mathbf{F}$ & . & . & $\mathbf{K}$ & . $\mathbf{M}$ & I $N$ & $\mathbf{O}$ & $\mathbf{P} \mathbf{Q}$ & $\mathbf{R}$ & . & $\mathbf{T}$ & . & $\mathbf{v}$ & . $\mathbf{x}$ \\
\hline$i \mathrm{M9}$ & & $\mathbf{C}$ & D & $\mathbf{E}$ & $\mathbf{F}$ & . & . & . & . & . & 0 & $\mathbf{P} \mathbf{Q}$ & $R$ & & . & . & . & . . \\
\hline$i \mathrm{M} 10$ & $\mathbf{B}$ & $\mathbf{C}$ & D & . & $\mathbf{F}$ & $\mathbf{H}$ & I. & $\mathbf{K}$ & . $\mathbf{M}$ & $\mathrm{IN}$ & 0 & . $\mathbf{Q}$ & $\mathrm{R}$ & $\mathbf{S}$ & . & . & . & \\
\hline$i$ M11 & B & $\mathbf{C}$ & D & $\mathbf{E}$ & . & . & . & $\mathbf{K}$ & L. & . & $\mathbf{O}$ & $\mathbf{P}$. & $\mathbf{R}$ & $\mathbf{S}$ & $\mathbf{T}$ & $\mathbf{U}$ & $\mathbf{v}$ & $\mathbf{w} \mathbf{x}$ \\
\hline$i \mathrm{M12}$ & B & $\mathbf{C}$ & D & $\mathbf{E}$ & $\mathbf{F}$ & . $\mathbf{H}$ & $\mathbf{I}$ & $\mathbf{K}$ & L $\mathbf{M}$ & $\mathbf{N}$ & $\mathbf{o}$ & $\mathbf{P} \mathbf{Q}$ & $2 \cdot$ & $\mathbf{S}$ & $\mathbf{T}$ & - & $\mathbf{V} \mathbf{v}$ & $\mathbf{W} \mathbf{x}$ \\
\hline rum detecting & $\begin{array}{l}135 \\
\text { and }\end{array}$ & & & & & & & & & & & & & & & & & \\
\hline
\end{tabular}

The activities on other mutant antigens of anti- $i$ (wild type) antiserum no. 133 and anti-iM13 antiserum no. 58 absorbed with different mutant antigens and pairs of antigens are shown in Table 4; see text for method of inference of factors from the data. The antigenic specificity common to the wild type and all the mutant antigens (factor $A$ ) is not shown. The anti-iM13 antiserum also detected a mutant specificity present in $i \mathrm{M4}(=i \mathrm{M5}=i \mathrm{M} 13)$ but absent from (wild type) and the other mutant antigens.

absent in $i \mathrm{M} 4, i \mathrm{M} 8$ and $i \mathrm{M} 11$. The sample absorbed with $i \mathrm{M} 4$ (which lacks $\mathrm{H}$ ) and with $i \mathrm{M} 6$ does not agglutinate $i \mathrm{M10}$, which has $\mathrm{H}$. We infer that absorption with $i \mathrm{M} 6$ has removed anti-H and that $\mathrm{H}$ is therefore present in $i \mathbf{M 6}$. A similar argument indicates that $i \mathrm{MI} 12$ also possesses factor $\mathrm{H}$. Antigens $i \mathrm{M} 7$ and $i \mathrm{M9}$ are unaffected by the samples containing anti-H and must therefore lack $\mathbf{H}$. The inferred distribution of $\mathrm{H}$ predicts that all samples not absorbed with $i \mathrm{M} 6, i \mathrm{M} 10$ or $i \mathrm{M} 12$ will agglutinate all three of these antigens; as was observed. Successive consideration of the remaining singly and doubly absorbed sera requires the postulation of factors $\mathbf{N}, \mathrm{L}, \mathrm{K}, \mathrm{M}, \mathrm{E}, \mathrm{B}, \mathrm{C}, \mathrm{F}$ and $\mathrm{D}$, all present in wild type and distributed amongst the mutants as shown in Table 4. The scheme accounts for all the positive and negative results in Tables 3 and 4; for every factor postulated there is at least one positive result, generally several, which cannot be otherwise accounted for, so that it seems unlikely that any unnecessary factors have been proposed. Absorption with any mutant antigen greatly decreased the titre of the anti- $i$ (wild type) serum on its homologous antigen. Since the anti-factor antibodies postulated are all of relatively low titre this implies that antibodies present in high concentration in the unabsorbed serum will combine with either the wild-type or with any mutant antigen. We assign the symbol $\mathbf{A}$ for the factor(s) common to the wild type and all the mutant antigens. 


\section{Attempt to map sites of amino acid substitution from serological results}

If each mutant antigen differs from the wild-type antigen by a single amino acid substitution it might be possible on certain assumptions to infer the sequence of the sites of amino acid substitution from the serological data. Suppose that each antigenic factor in the wild-type antigen (i.e. each chemical feature recognized by a specific family of antibody molecules in an anti- $i$ antiserum) was completely defined by a given sequence of amino acids in the $i$ flagellin polypeptide chain, and that each anti-factor antibody had an absolutely specific affinity for all the amino acids in the corresponding sequence (or at least for all of a class of serologically important amino acids within it). The $i$ flagellin and $i$ antigen could then be represented by a single straight-line map of the amino acid sequence, with every wildtype factor shown by a single line covering a certain number of the amino acid sites. A serologically altered mutant flagellin resulting from a single amino acid change ex hypothesi would not combine with any anti-factor antibody with affinity for a sequence of amino acids which included the site of substitution, and the mutant antigen would therefore lack the corresponding wild-type factors. On such a map, and a forteriori on a simplified map, showing only in their correct sequence the sites of amino acid substitution of a series of mutants, all the wild-type factors absent from a given mutant would be represented by unbroken lines of appropriate length, all spanning the site of substitution of that mutant, and some of them spanning also one or more adjacent substitution sites, on one or both sides, corresponding to the other mutants which lacked the wild-type factor represented by the line in question. On the assumption that the eight serologically distinct mutant antigens each arose by a single amino acid substitution we tried to arrange the eight hypothetical substitution sites in a linear order such that all the wild-type factors inferred as absent from one or more mutants could be represented as described above. However, no order permitted representation of each missing factor by an unbroken line. Some factors of $i$ might comprise the $\mathrm{C}$-terminus of one flagellin molecule and the $\mathrm{N}$-terminus of an adjacent molecule (cf. the antigenic factor present in tobacco mosaic virus (TMV) and re-aggregated TMV A-protein but absent from free TMV A-protein, Aach, 1959). The linear map of the antigen and its factors would then be a circle instead of a straight line. The missing factors of the mutants could not, however, be represented by single lines on such a map.

On the above assumptions the order of the sites of substitution in a series of mutant antigens could also be inferred directly from the activity or inactivity on other mutant antigens of anti-wild-type serum fully absorbed with different pairs of mutant antigens. Consider a mutant antigen, $P$, with a substitution at site $p$ in the polypeptide chain and another mutant antigen, $Q$, with a substitution at site $q$. Absorption of an anti-wild type antiserum with antigens $\boldsymbol{P}$ and $\boldsymbol{Q}$ would leave only antibody with affinity for amino acid sequences which spanned both site $p$ and site $q$. These residual antibodies would ex hypothesi have affinity for all the antigenically important sites between $p$ and $q$ in the wild-type antigen, and would therefore fail to agglutinate a third mutant, $R$, if its site of substitution, $r$, were between sites $p$ and $q$. Thus if antiserum absorbed with mutant antigens $\boldsymbol{P}$ and $\boldsymbol{Q}$ agglutinated mutant antigen $R$ then, on the assumptions stated, the order of the sites of their amino acid changes cannot be $p-r-q$. The data of Table 4 were therefore analysed 
in respect of each possible set of three mutant antigens, drawn from the eight tested. For each set there were available observations on three different doubly absorbed samples of anti-wild-type antiserum, each capable of excluding one of the three possible orders. None of the 56 sets gave the self-contradictory result (all three orders excluded); two orders were excluded in 21 sets, one order in 30 sets and no order in the remaining 5 sets. However, no sequence of the eight sites of amino acid substitution was found which would satisfy all 21 of the unequivocally indicated orders within sets of three. A few discrepant results remained even when it was assumed that mutants $\mathrm{M} 7$ and $\mathrm{M} 9$, concerned in most of the contradictions, were double mutants, each having two different sites of amino acid substitution.

\section{Analysis of wild-type antigen $i$ by absorption of an anti-mutant serum}

In view of the complexity of the wild-type antigen $i$ revealed by the activities on the mutant antigens of an anti- $i$ (wild type) antiserum absorbed by different mutant antigens and pairs of antigens (Tables 3,4) it was decided to make a similar investigation with an anti-mutant antiserum. Anti- $i$ M 13 antiserum no. 58 was used; after complete absorption with wild-type antigen this antiserum agglutinated only its homologous antigen, $i \mathrm{M} 13$ (and the serologically identical antigens $i \mathrm{M} 4$ and $i$ M5) (Table 2). Samples fully absorbed with each of the different mutant antigens other than the homologous antigen and with each pair of such mutant antigens were tested qualitatively on the wild-type and mutant antigens (Table 4). As was expected all the absorbed samples agglutinated the homologous suspension; as this reflected the presence of mutant-specific antibodies (Table 2) these reactions are omitted in Table 4. The activities of the absorbed samples of the anti-iM13 antiserum on mutants other than $i \mathrm{Ml} 13$ (Table 4) must have resulted from the presence of antibodies active on factors present in the wild-type antigen (since absorption with wild-type antigen removed all activity except that on the homologous antigen (Table 2)), and present also in antigen $i \mathrm{M13}$, used for immunization and capable of removing all activity from the antiserum. It was therefore expected that the activities on the mutant antigens of the absorbed anti-iM13 antiserum would be similar to those of the absorbed anti- $i$ (wild-type) sera (Tables 3 and 4), except for the absence of the positive reactions attributed to antibodies against the wild-type factors $\mathrm{D}, \mathrm{G}, \mathrm{H}, \mathrm{J}, \mathrm{N}$, inferred to be absent from antigen $i \mathrm{M13}$ (Table 5). Most of the reactions obtained agreed with this expectation. Some of the absorbed antimutant samples, however, were active on mutant antigens unaffected by the corresponding sample of anti- $i$ (wild type) antiserum. Some such differences were apparent when the singly absorbed antisera were compared; for instance, the anti$i$ M13 antiserum absorbed with $i$ M10, but not the similarly absorbed anti- $i$ (wild type) antiserum, strongly agglutinated antigens $i \mathrm{Mr}$ and $i$ M8. As four different anti- $i$ (wild type) antisera after single absorption with different mutant antigens gave qualitatively identical results on the remaining mutant antigens (Table 3), it seems unlikely that the unexpected additional activity of some absorbed samples of anti$i \mathrm{M} 13$ antiserum no. 58 reflected merely chance differences in the response of different rabbits to minor factors in antigens $i$ (wild type) and $i \mathrm{M} 13$.

The data of Table 4 were analysed to find the minimum number of antigenic factors present in both wild-type $i$ and $i \mathrm{M} 13$ antigens which would account for the observed activities of the absorbed samples of anti-iM13 antiserum, by using the 
same kind of argument as was used for analysis of the activity of absorbed samples of anti- $i$ (wild-type) antiserum no. 133. The results are recorded in Table 5. Of the seven wild-type factors previously inferred to be present in antigen $i \mathrm{M13}$, only factors $\mathrm{B}$ and $\mathrm{C}$ were recognizable by analysis of the activities of the anti- $i \mathrm{M} 13$ antiserum. It was necessary to postulate an additional ten factors ( 0 to $\mathrm{X}$ in Table 5), each present in the wild-type and $i \mathrm{M} 13$ antigens and each with a distinctive distribution amongst the other mutant antigens.

\section{DISCUSSION}

Analysis of a serum prepared against the wild-type phase-1 antigen $i$ of Salmonella typhimurium strain LT 2 by absorption and agglutination tests with mutant forms of this antigen showed that many different antigenic subfactors can be recognized in the wild-type antigen. The single symbol $i$ recorded in the KauffmannWhite table for the phase-1 antigen of many species perhaps conceals some variation in the naturally occurring antigen. The phase-1 antigen $i$ of $S$. landau is known to differ somewhat from the standard form (Günther \& Hauser, 1954) and in the original descriptions of many other species the complete identity of the phase-1 antigen with standard antigen $i$ was not demonstrated, since 'mirror' absorption tests were not made. It would not be surprising if some of the mutant forms of antigen $i$ were to occur as the wild-type phase-1 antigen in some species other than $S$. typhimurium (or in some wild strains of this species); the previously undescribed antigen $l, z_{40}$ of $S$. rutgers was found to be closely related to an unnamed mutant form of antigen $l, v$ previously obtained in the laboratory by serum selection (Drs P. R. Edwards \& D. W. Bruner, personal communication).

All the eight serologically distinct mutant antigens had lost some of the serological specificity of the wild-type antigen and were agglutinated to rather lower titres than it by the anti- $i$ (wild-type) antiserum used in their isolation-as was to be expected since they were detected by their ability to spread more rapidly in the presence of this serum. One wild-type specificity (factor G, Table 5) was missing from all the mutant antigens; but each of the eight serologically different antigens also lacked a different combination of wild-type factors. Serological mutants were easily obtained by selection with serum 713, either unabsorbed or absorbed with $i 2(=i \mathrm{M} 8)$, the first mutant antigen to be isolated. This perhaps indicates that in this antiserum the antibody with the highest immobilization titre was specific for a factor or factors easily lost by mutation. Later attempts to isolate serological mutants by selection with other anti- $i$ sera failed.

On inoculation into rabbits all the mutant antigens (except perhaps the nonspecifically agglutinable $i \mathrm{M} 6$ and $i \mathrm{M} 9$ ) evoked some antibody unable to combine with wild-type antigen. Perhaps any alteration in the amino acid sequence of an antigenically determinant part of the flagellin molecule (or at least ahy change in a serologically important amino acid in this sequence) results in the evocation of antibody molecules with affinity for the new features, presumably amino acid sequences, absent in the wild-type molecule. Rather similarly tobacco mosaic virus (TMV) from which the carboxy-terminal threonine of the constituent protein has been removed by digestion with carboxypeptidase differs from native TMV by the absence of one 'native' serological specificity and the appearance of a new specificity 
(Harris \& Knight, 1955). The mutant specificities common to $i \mathrm{M} 7, i \mathrm{M} 10$ and $i \mathrm{M} 12$ (Table 2) are unexpected if, as we suppose, each of these mutant antigens differs from the wild type by a single amino acid substitution at a different site. Possibly each of the three different substitutions causes a similar change in the tertiary structure of the $i$ flagellin molecule, and thereby produces a similar new feature (e.g. the approximation of two antigenically important amino acid side-chains) in a part of the molecule distant from the site of amino acid substitution.

We do not know the cause of the curious agglutinability of $i \mathrm{M} 6$ and $i \mathrm{M} 9$ suspensions by the sera of non-immunized rabbits, and, to higher titres, by the sera of rabbits inoculated with related or unrelated antigens, even after complete absorption with the immunizing antigens. The flocculation of indian ink by these antigens suggests that a change in some physical property of the flagellar protein may be concerned; but, contrary to what would be expected if they were agglutinable by an exceptionally small amount of antibody, these antigens were not agglutinated to higher titres than other antigens by various anti- $i$ (mutant or wildtype) sera (Tables 1 and 2).

In all the mutants the site of mutation is in $H 1$, the structural gene for phase-1 flagellin (Joys \& Stocker, 1963, and unpublished results). In four of the mutant flagellins a single change in the tryptic or peptic peptide 'map' has been detected (McDonough, 1962). It is reasonable to assume that in all the mutant antigens the difference from the wild type consists in a single amino acid substitution. On this assumption it follows that a single amino acid change can destroy the affinity of the wild-type antigen for several different families of antibody (since each mutant antigen has lost several different wild-type antigenic factors); this suggests that the antibodies evoked by a flagellar antigen include very many species, some with affinities for overlapping features of the antigenically determinant part of the flagellum. The failure of our attempt to discover the order of the sites of amino acid substitution in the different mutants, either by consideration of the factors inferred to be missing in different mutant antigens (Table 5) or directly from the activity or inactivity on the remaining mutant antigens of anti-wild-type antiserum absorbed with pairs of mutant antigens (Table 4), indicates the incorrectness of one of the assumptions involved-probably the assumption that every antigenic factor consists of a length of polypeptide chain and that change of any amino acid (or of any antigenically determinant amino acid) in this length would result in complete loss of affinity for the anti-factor antibody concerned. It is likely that some anti-protein antibodies are specific for a given short sequence of amino acids, regardless of their configuration in the complete antigen; for instance, an artificial antigen consisting of a carrier protein to which is attached a synthetic oligopeptide comprising the last $6,5,4$ or 3 amino acids at the carboxy terminus of TMV protein evokes antibody which precipitates and neutralizes TMV (Anderer \& Schlumberger, 1965). But it may well be that some species of antibody molecules have specificities for pairs of chemical features which are close together on the surface of the flagellum but far apart along the flagellin polypeptide chain; the existence of such antibodies in the anti- $i$ antiserum examined would invalidate the attempted analysis. It is also possible that some of the serological changes caused by the mutations reflect not alterations in amino acids whose side-chains are themselves part of an antigenically determinant surface, but alterations of the configuration of the antigenically determinant part 
of the flagellin molecule. However, as all the mutant flagellins support normal locomotor function it is unlikely that the amino acid substitutions cause any profound change in the tertiary structure of the flagellin molecule, at least within the complete flagellum.

In microbial genetics most mutations involving alteration of the structure of a protein are recognized by a defect in some function, which may reflect either the absence of a recognizable protein product, e.g. enzyme, or the production of an abnormal protein. Various abnormal enzymic proteins are recognized as crossreactive material (CRM) by their reaction with antibody evoked by the wild-type antigen. In general the CRM was not distinguished serologically from the wild-type protein by the techniques employed; the use of quantitative complement fixation methods has shown that some mutant tryptophan-synthetase proteins of Neurospora crassa cross-react with, but are serologically distinguishable from, the wildtype enzyme, and has disclosed also a correlation of site of mutation within the structural gene with kind of alteration in serological character (Kaplan, Mills, Ensign \& Bonner, 1964). The flagellar antigen of Salmonella typhimurium, though it lends itself exceptionally well to detailed serological analysis in terms of antigenic subfactors, is somewhat intractable material for fine-structure genetical mapping; and the sequence of the $\mathbf{3 8 0}$ or so amino acids in the molecule (McDonough, 1965) is as yet unknown. It is therefore unlikely that the mutational alterations in serological character described above will be interpretable in the near future by reference to a complete genetical and amino acid sequence map, such as is available for the tryptophan-synthetase proteins of Escherichia coli (Yanofsky et al., 1964).

\section{REFERENCES}

АACH, H. G. (1959). Serologische Untersuchungen zur Struktur des Tabakmosaikvirus. Biochim. biophys. Acta, 32, 140.

Anderer, F. A. \& Schlumberger, H. D. (1965). Properties of different artificial antigens related to tobacco mosaic virus. Biochim. biophys. Acta, 97, 503.

Arkwright, J. A. (1927). Microscopic evidence for the different manner of clumping of motile bacteria with somatic and flagellar agglutinins. J. Path. Bact. 30, 566.

Bruner, D. W. \& Edwards, P. R. (1939). A note on the monophasic Salmonella types. J. Bact. 37, 365.

Craigie, J. (1931). Studies on the serological reactions of the flagella of B. typhosus. J. Immunol. 21, 417.

Edwards, P. R. \& Bruner, D. W. (1939a). Reversibility of the alpha and beta phases of Salmonella typhi. Proc. Soc. exp. Biol. Med. 41, 223.

Edwards, P. R. \& Bruner, D. W. (1939b). The demonstration of phase variation in Salmonella abortusequi. J. Bact. 38, 63.

EdWARDs, P. R. \& BRUNER, D. W. (1942). Serological identification of Salmonella cultures. Circ. Ky agric. Exp. Stn no. 54.

GARD, S. (1937). Das Schwärmphänomen in der Salmonella-Gruppe und seine praktische Ausnützung. Z. Hyg. InfectKrankh. 121, 139.

Gnosspelius, A. (1939). Über künstlisch Veränderungen des H-Antigens in der Salmonella-Gruppe. Z. Hyg. InfectKrankh. 121, 529.

GüNTHER, O.\& HAUSER, A. (1954). Salmonella $30: \mathbf{i} ; 1,2$ : ein neuer Salmonellatyp. Zentbl. Bakt.ParasitKde (Abt. 1. Orig.), 161, 363.

Harris, J. I. \& KNIGHT, C. A. (1955). Studies on the action of carboxypeptidase on tobacco mosaic virus. J. biol. Chem. 214, 215.

Joys, T. M. (1961). Mutation of flagellar antigen $i$ in Salmonella typhimurium. Ph.D. Thesis, University of London. 
Joys, T. M. \& Stocker, B. A. D. (1963). Mutation and recombination of flagellar antigen $i$ of Salmonella typhimurium. Nature, Lond. 197, 413.

Kaplan, S., Mills, S. E., Ensign, S. \& Bonner, D. W. (1964). Genetic determination of the antigenic specificity of tryptophan synthetase. J. mol. Biol. 8, 801 .

Kauffmann, F. (1951). The Enterobacteriaceae, 1st ed. Copenhagen: Ejnar Munksgaard.

Lederberg, J. \& Edwards, P. R. (1953). Serotypic recombination in Salmonella. $J$. Immunol. 71, 232.

McDonough, M. W. (1962). Tryptic peptide maps of mutant Salmonella flagellins. Biochem. J. 84, 114 P.

McDonougr, M. W. (1965). Amino acid composition of antigenically distinct Salmonella flagellar proteins. J. mol. Biol. 12, 342.

Mackie, T. J. \& MCCantney, J. E. (1953). Handbook of Practical Bacteriology, 9th ed. Edinburgh: Livingstone.

Mäkelä, P. H. (1964). Genetic homologies between flagellar antigens of Escherichia coli and Salmonella abony. J. gen. Microbiol. 35, 503.

Mandelbaum, M. (1932). Zur Typendifferenzierung innerhalb der Typhus-Paratyphusgruppe durch neue serologische Methoden und die Anwendung derselben zur Diagnose der durch diese Keime verusachten Krankheiten. Zentbl. Bakt.ParasitKde (II. Ref.), 105, 377.

Scotr, W. M. (1926). The 'Thompson' type of Salmonella. J. Hyg., Camb. 25, 398.

Smith, S. M. \& Stocker, B. A. D. (1962). Colicinogy and recombination. Br. med. Bull. $18,46$.

Stocker, B. A. D. (1956). Abortive transduction of motility in Salmonella : a non-replicated gene transmitted through many generations to a single descendant. J. gen. Microbiol. 15, 575 .

Stocker, B. A. D., McDonough, N. W. \& Ambler, R. P. (1961). A gene determining presence or absence of $\epsilon-N$-methyl-lysine in Salmonella flagellar protein. Nature, Lond. $189,556$.

Stocker, B. A. D., Zinder, N. D. \& Lederberg, J. (1953). Transduction of flagellar characters in Salmonella. J. gen. Microbiol. 9, 410.

Yanofsky, C., Carlton, B. C., Guest, J. R., Helinski, D. R. \& Henning, U. (1964). On the co-linearity of gene structure and protein structure. Proc. natn. Acad. Sci. U.S.A. 51, 266.

Zinder, N. D. \& Lederberg, J. (1952). Genetic exchange in Salmonella. J. Bact. 64, 679. 